\title{
There is no spoon: applying virtual reality for maintenance training of rolling stock technicians
}

\author{
Alberto Martinetti ${ }^{*}, \mathrm{~K}$. 't Hart and \\ Roy Damgrave
}

Design, Production and Management Department, University of Twente, The Netherlands

Email: a.martinetti@utwente.nl

Email: koentehart@gmail.com

Email: r.g.j.damgrave@utwente.nl

*Corresponding author

\section{L.A.M. van Dongen}

Design, Production and Management Department, University of Twente, The Netherlands and

Netherlands Railways, The Netherlands

Email: 1.a.m.vandongen@utwente.nl

\section{R. Turkenburg and Andre Nouwens}

Netherlands Railways, The Netherlands

Email: robin.turkenburg1@ns.nl

Email: Andre.Nouwens@NS.NL

\begin{abstract}
Introducing into operations new rolling stocks requires re-educating technicians and operators for being able to properly work on new and different assets. The training sessions are often performed under substantial time constraints and with a lack of resources. Re-training the technicians is a cost intensive process as it is for training new employees. The aim of the paper is to test and evaluate a virtual reality (VR) training solutions to decrease costs and to increase technicians' availability, safety during maintenance operations and education performance with the support of Netherlands Railways (Nederlandse Spoorwegen - NS). Firstly, the study explores the possible maintenance tasks analysing the benefits for VR implementations; secondly, it evaluates the attitude of technicians trained with the proposed VR approach. Finally, it discusses the results and provides general applicable suggestions for the use of VR in other training activities.
\end{abstract}

Keywords: maintenance; virtual reality; VR; rolling stocks; training. 
Reference to this paper should be made as follows: Martinetti, A., 't Hart, K., Damgrave, R., van Dongen, L.A.M., Turkenburg, R. and Nouwens, A. (2018) 'There is no spoon: applying virtual reality for maintenance training of rolling stock technicians', Int. J. System of Systems Engineering, Vol. 8, No. 4, pp.398-415.

Biographical notes: Alberto Martinetti is an Assistant Professor in Maintenance Engineering at the University of Twente. He is the Coordinator of the Maintenance Engineering and Operations (MEO) specialising in Mechanical Engineering. He has worked for the Polytechnic of Turin and for the University of Turin. He holds a Master's degree in Geo-resources and Geo-technologies Engineering and a PhD degree in Safety and Health from the Polytechnic of Turin (2013). His expertise and research interests are occupational safety and health, maintenance engineering and the application of new technologies to maintenance and safety domains.

K. 't Hart is a Mechanical Maintenance Engineer at the Gunvor Petroleum Rotterdam B.V. specialising in maintenance and implementing/adapting theoretical maintenance policies for possible benefits. He holds a degree in Mechanical Engineering at the University of Twente.

Roy Damgrave graduated in Industrial Design Engineering at the University of Twente in the specialisation Management of Product Development, on the subject of using motion capturing in product development processes. Since 2008, he works as an Assistant Professor at the Laboratory of Design, Production and Management, part of the Engineering Technology Faculty of the University of Twente. His research is mainly focused on the use of virtual and augmented reality in product development processes. In June 2017, he received his $\mathrm{PhD}$ at the University of Twente on the subject enhancing the effectiveness of design tools in synthetic environment.

L.A.M. van Dongen has worked for the Netherlands Railways (NS, 100\% state owned) since 1984. Since 2016, he holds the position of Chief Technology Officer of NS. Since 2010, he has been a part-time Professor in Maintenance Engineering at the University of Twente. He completed his doctoral research at the Eindhoven University of Technology on energy efficiency of drive trains for electric vehicles. His career within NS has concentrated mainly on technological functions (electric locomotives, fleet manager) and now he is responsible for technical fleet management, maintenance systems, spare parts purchase, maintenance management and construction of new capital goods workshops.

R. Turkenburg is a Manager of the Train Test Centrum at NS leading a team of 40 professionals who ensure the correct performance, safety and reliability of (new) train equipment methodically tested.

Andre Nouwens is the Director of Material Engineering Development, Talent and Organisation Development and Course Development at the Netherlands Railways. He is also Director for supplying efficient and effective (learning) interventions to enable employees to learn, maintain and improve existing and new professional knowledge/skills. 


\section{Introduction}

Maintenance changed from an obligatory part of running a business to strategic element, from being seen as a 'necessary evil', 'technical matter', to a 'pro contributor' and finally to a 'cooperative partnership'. Moreover, this transformation goes hand in hand with the ever-increasing complexity of assets (Pintelon and Parodi-Herz, 2008).

Maintenance is undoubtedly a critical success factor (Rajabalinejad et al., 2016) for ensuring a high-quality railway service and the tasks are complex operations that require a relevant amount of information and experience (Martinetti et al., 2017). It is dependent on the expertise of its technicians and high skilled technicians can increase the efficiency of maintenance nowadays.

The introduction of new assets into operations requires a re-training or a re-education of the technicians. The training sessions are performed, often, under substantial time constraints and with a lack of resources. At the same time, the new rolling stocks have to be implemented within the existing operational schedule. It generally means a limited time for the training sessions on the new assets.

Within the Dutch railway network, the maintenance operations can take place at several locations throughout the Netherlands.

On-location training is often unfeasible due to limited access to the train or due the time requested for transporting the technicians to the training location, lowering the efficiency of the training. Secondly, the technician requires on average (at least) two days of practical training during which the asset is unavailable for service. The consequence is that re-training the technicians is a cost intensive process comparable with training new employees.

The research has been performed with the support of Netherlands Railways (Nederlandse Spoorwegen - NS) for investigating the benefit of applying VR during the introduction of the new Fast Light Regional Trains (FLIRT) (Figure 1).

Figure 1 Fast Light Regional Trains in Maastricht service station (see online version for colours)

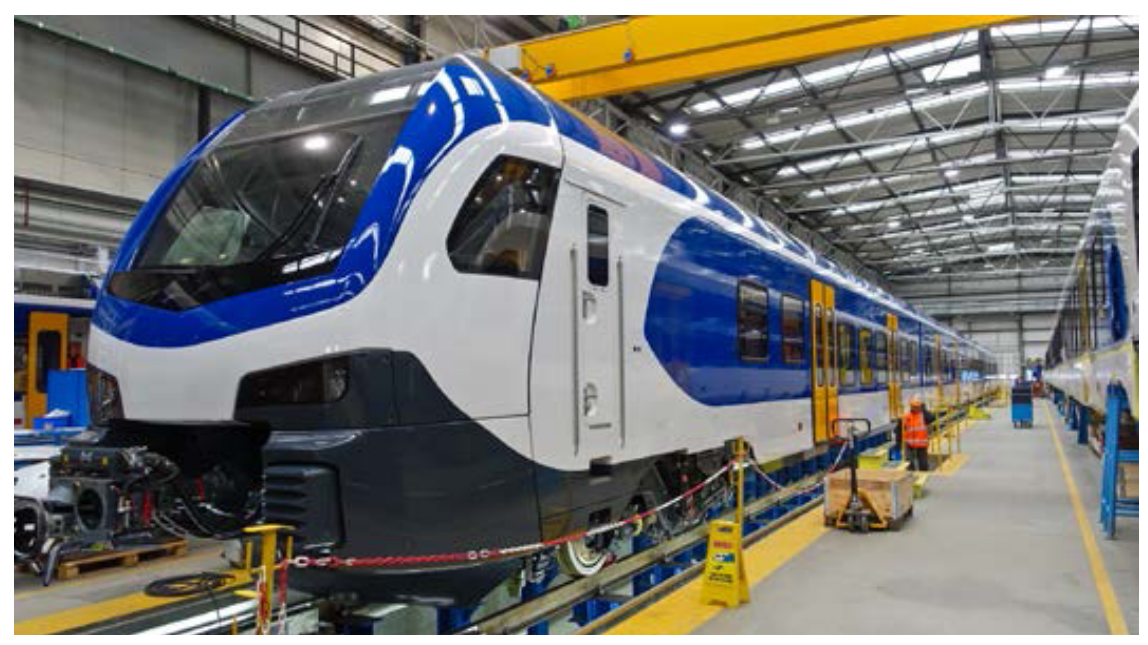

The paper studies the value (in terms of increased learning retention, performance, and reduced errors) of using VR to train technicians for a critical task during the maintenance operations of the FLIRT. The proposed solution aims to decrease costs and time of 
maintenance operations and to increase safety during maintenance operations and education effectiveness.

The technicians are introduced to a virtual environment wherein the components to be studied are reproduced. Being immersed in the VR environment, the technicians can interact with the components and the interactivity is achieved without any hand-held devices.

Figure 2 Flowchart presenting the main parts of the research (see online version for colours)

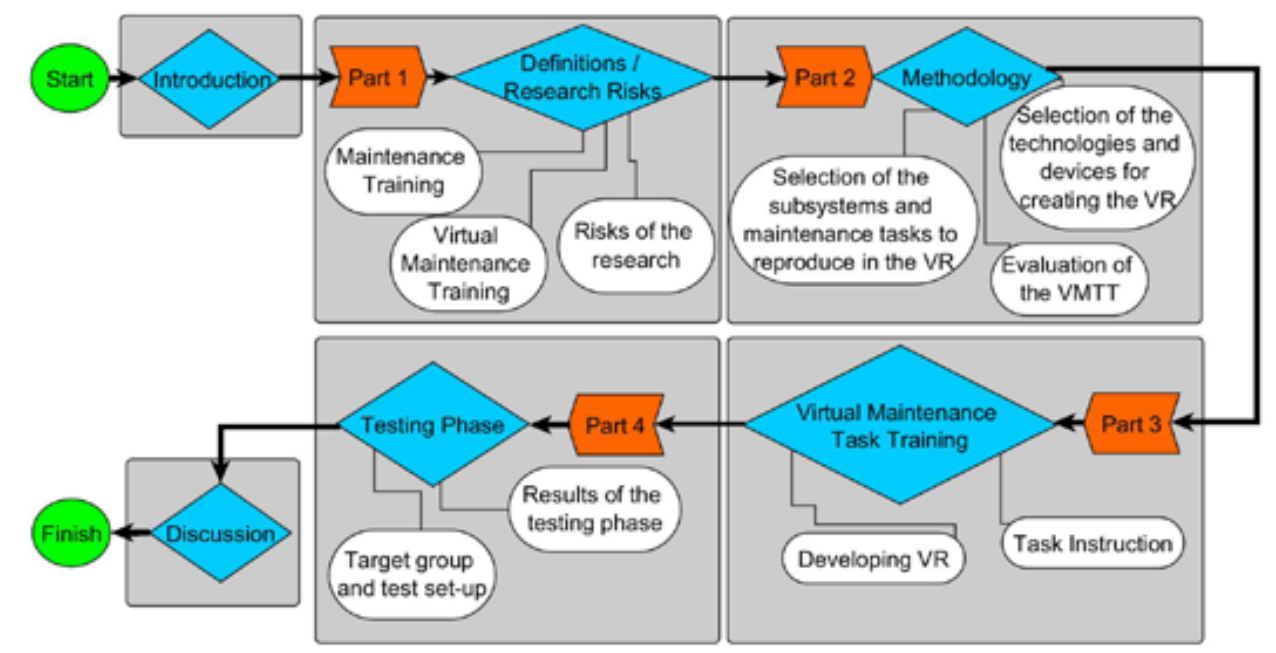

The research consists of four main parts (Figure 2). Firstly, in part 1 the definitions of the actual Maintenance Training Programme of the NS and of the possible VR training is, highlighting possible risks involved in the research. Secondly, part 2 offers an overview of the methodology, describing the evaluation method and the selected maintenance operations to reproduce in the virtual environment and the related motivations.

After that, part 3 explains the creation of a virtual maintenance task training (VMTT) highlighting the products capabilities and limitations. Finally, part 4 reports the results obtained during the testing phase of the virtual training. The final discussion paragraph of the paper presents future steps and reflections on the virtual system.

\section{Part 1-Definitions and risks of the research}

\subsection{Maintenance training}

To ensure the technicians have the required maintenance expertise the European Federation of National Maintenance Societies (EFNMS) developed specifications for this knowledge (EFNMS, 1998). The aim of the certifications is to create technicians that can prove their competence and become certified European experts in maintenance. The qualifications are based on the European Credit Transfer and Accumulation System (ECTS) and thus they are transferable between countries and international companies.

As discussed by Salas et al. (2012), to offer a proper training several measures have to be taken before, during, and after training in order to respectively have: 
- clear schedule with an attendance policy and well-prepared supervisors before the training

- structured instructions, opportunities to practice, and opportunities for making mistakes during the training

- assessment of the training and of the gained knowledge after the training.

Due to the fast learning decay of the maintenance procedures (usually characterised by long list of steps to memorise), an immediate use of the trained knowledge is required. As highlighted by Ebbinghaus (2013), the application of gained knowledge is critical, and crucial, to the retention of the contents. Since the technicians have to assure that assets remain reliable and safe at the same time, the training of the technicians is a primary concern for the NS in the strategic maintenance management.

NS has a clear education structure to provide different levels of expertise for the technicians based on a blended learning approach and on the European Qualification Framework (EQF). According to Bonk et al. (2012), the blended learning style limits the use of a formal education program, it minimises the need for educators and lecture rooms and it trains technicians from an operational point of view at an early stage.

After a vocational training to acquire knowledge on mechanical, electrical, electronic and control systems (EQF 1), the technicians are trained for having a basic learning on inspection and maintenance (EQF 2), for having a basic learning on fault finding and connection (EQF 3) and for having a basic learning on specific electronics and high voltage component assembly (EQF 4).

\subsection{Virtual maintenance training}

Bamodu and Ye (2013) defines VR as a "high-end human-machine interface, which combines technologies such as computer graphics, image processing, pattern recognition, artificial intelligence, networking, and sound systems to produce computer simulations and interactions."

Therefore, a virtual maintenance training (VMT) uses interactive 3D simulations to explain how to maintain assets. It creates a VR environment wherein the user, in the case study, the trainee, can experience several scenarios to test his/her skills. The trainee can inspect and repair failed components within the VR to restore the system. With Input and Output (IO) devices, the trainee can interact with the system and apply the necessary procedures to complete the scenarios.

In recent years, a better quality of technical documentations and an increase of computer processing power have resulted in better results in applying VMT, producing realistic digital components.

The VMT offers several advantages. A first benefit is the opportunity to be less dependent from the availability of the asset for training purposes. The training can take place without reserving a costly asset, classroom, or supervision. Secondly, due to the aging of the workforce and its consequent retirement, the companies have to face a growing lost in knowledge. The VMT's application can retain this knowledge transferring it to new technicians. 


\subsection{Risks related to the VMT}

Moving away from traditional training methods could bring several risks that could affect the quality of the training (i.e., the learning retention or the technical and procedural competences). Consequently, the training design has to be carefully planned (re-thinking the actual learning framework) for ensuring a proper balance between the level of details of VR environment and the useful information to transfer to the technicians.

Moreover, financial risks concerning the implementation of VR have to be taken into account. An initial large investment is usually required for buying the devices and for acquiring the $\mathrm{CAD}$ files owned by the manufacturers of the assets.

Finally, there are technological risks involved in the process of creating the digital representation of the train. The major risks could be related to the source files, to the VR environment or to the IO devices. The source files require compatibility with various softwares. Close cooperation with the OEM is required to fit the output of file extensions with the preferred input of the VR creation process. Moreover, the environment needs constant updating not only for changing the digital components but also for readjusting the work instructions. The upkeep of these updates could be very time-consuming. For a mobile application, this may be up to $20 \%$ of the total application development costs (Chomko, 2012).

\section{Part 2 - methodology}

The VMTT provides a platform for technicians to digitally experience work instructions. It allows them to study components, locations, and procedures in a safe environment.

The research has been structured as follows:

- selection of the subsystem and of the maintenance task to reproduce in the VR

- selection of the technology and of the devices for creating the VR

- evaluation of the VMTT.

\subsection{Selection of the subsystems and maintenance tasks to reproduce in the VR}

As mentioned in the introduction, the train interested by the research is the FLIRT. The train developed by Stadler, can reach a top speed of $160 \mathrm{~km} / \mathrm{h}$ and, depending by number or couches, transporting up to 336 commuters (Stadler Bussnang AG, 2016). It is electrically driven and purposefully designed for regional transport demanding only qualified personnel to operate, inspect, and maintain the train due to its complexity.

The selection of the subsystem and of the consequent maintenance task to reproduce is driven by two main points of interest:

- frequency of service requests (in order to test relevant tasks from a failure criticality perspective)

- learning issues and feasibility from a technological perspective (the assembly cannot consist of too many components, with too extended maintenance procedures). 
Since the introduction of the FLIRT, several service requests arrived to the NS for corrective maintenance actions.

Figure 3 The service request per component in percentages (see online version for colours)

\section{Percentage of Service Request per Component}

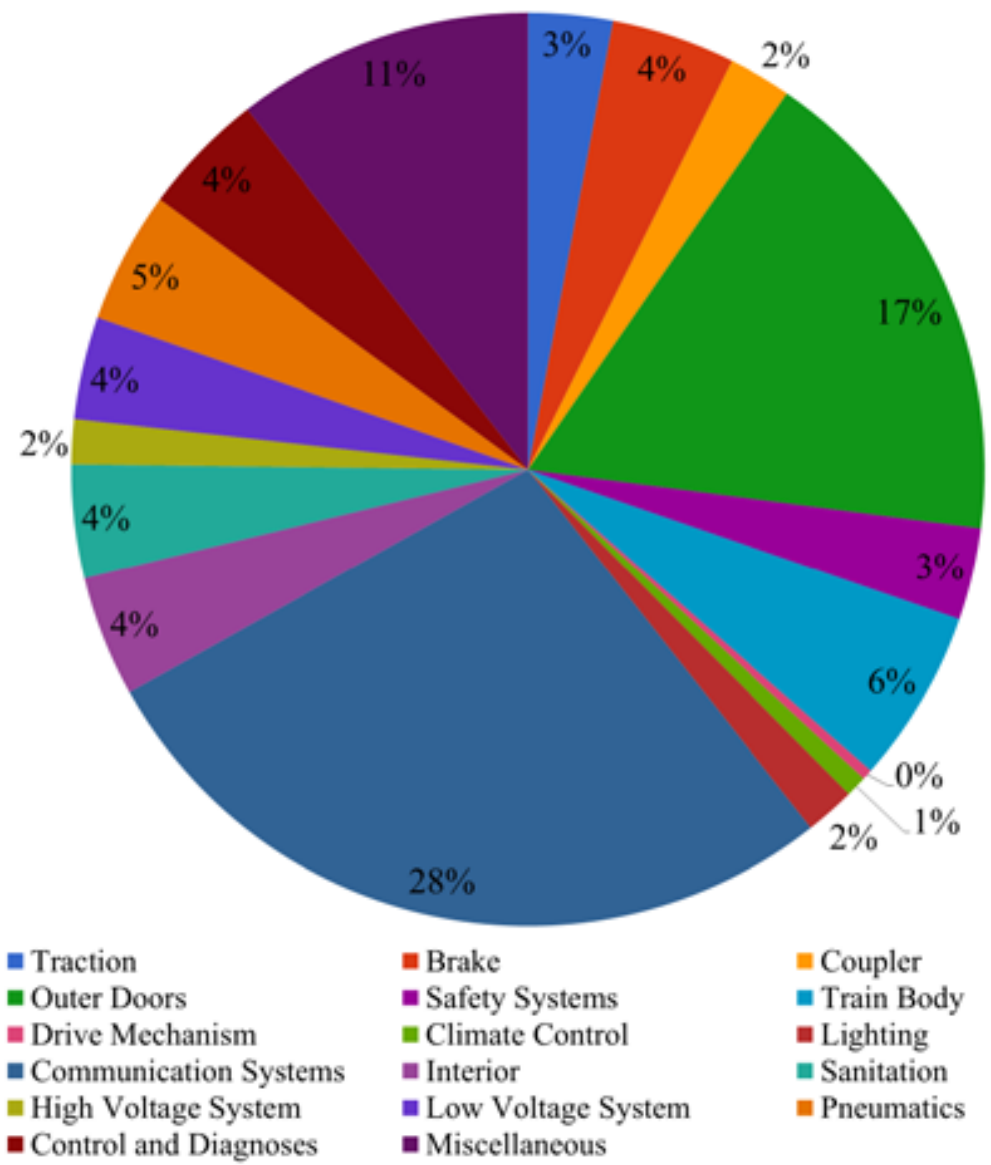

As shown in Figure 3, amongst all the requests, the door subsystem accounted for 17.4\%, only surpassed by the communications system of the train (28\%).

Because of the nature of the communications system (digital), most of the failures result in a computer problem, usually solved with a system reboot without the need of replacing parts or disassembling the system.

Finally, the door system has an intrinsic failure criticality: if a failure happens forcing the doors to stay open, the train is, by law, not allowed to continue driving.

According to those reflections (and after several discussions with the NS team responsible for the introduction of the FLIRT into the Dutch railway network), the door motor subsystem has been selected and considered the best case study (Figure 4) for testing the VMMT. 
Figure 4 The service request per component in percentages (see online version for colours)

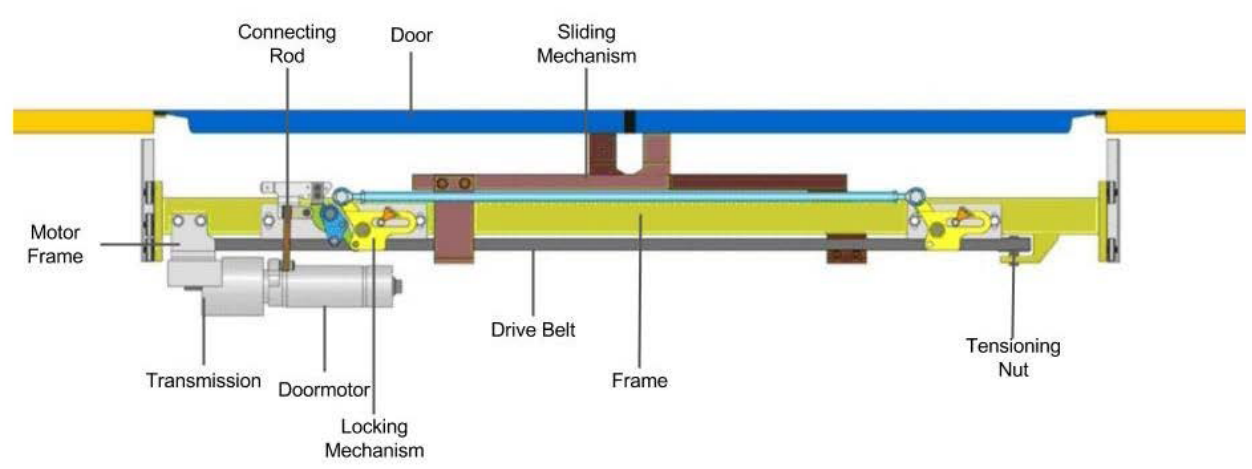

Table 1 shows the main steps of the procedure (the preparation, the disassembly, the re-assembly and the conclusion) for replacing the door motor.

Table 1 Steps for maintain the door motor subsystem

\begin{tabular}{|c|c|c|c|}
\hline \multicolumn{2}{|c|}{ Preparation } & \multicolumn{2}{|r|}{ Door motor disassembly } \\
\hline 1 & Turn off electrical system & 1 & Loosen the drive belt \\
\hline \multirow[t]{5}{*}{2} & \multirow{5}{*}{$\begin{array}{l}\text { Open hatch to uncover door } \\
\text { mechanism }\end{array}$} & 2 & Remove connecting \\
\hline & & 3 & Remove door motor assembly \\
\hline & & 4 & $\begin{array}{l}\text { Remove seeger ring, sprocket, and } \\
\text { frame }\end{array}$ \\
\hline & & 5 & Remove transmission \\
\hline & & 6 & Remove door motor \\
\hline \multicolumn{2}{|c|}{ Door motor re-assembly } & & Conclusion \\
\hline 1 & Place door motor & 1 & Turn on electrical system \\
\hline 2 & Place frame, sprocket and seeger ring & 2 & Test function of the doors \\
\hline 3 & Place door motor assembly & 3 & Close hatch \\
\hline 4 & Place connecting rod & & \\
\hline 5 & Tighten drive belt & & \\
\hline
\end{tabular}

\subsection{Selection of the technology and of the devices for creating the VR}

The VR is supported by the adoption of the Leap Motion technology for tracking the body movements of the technicians and by the Head-Mounted Display (HMD) Oculus (Figure 5) for displaying VR environment.

Unity3D has been adopted as 3D modelling software due to its widespread availability and its full compatibility with Javascript and C\# integration. Furthermore, it is capable of exporting to multiple platforms including, but not limited to, PC, Mac OS $\mathrm{X}$, and other applications. 
Figure 5 Set-up of the workspace with the leap motion sensor combined with the HMD oculus DK2 during the testing phase in Maastricht service location (see online version for colours)

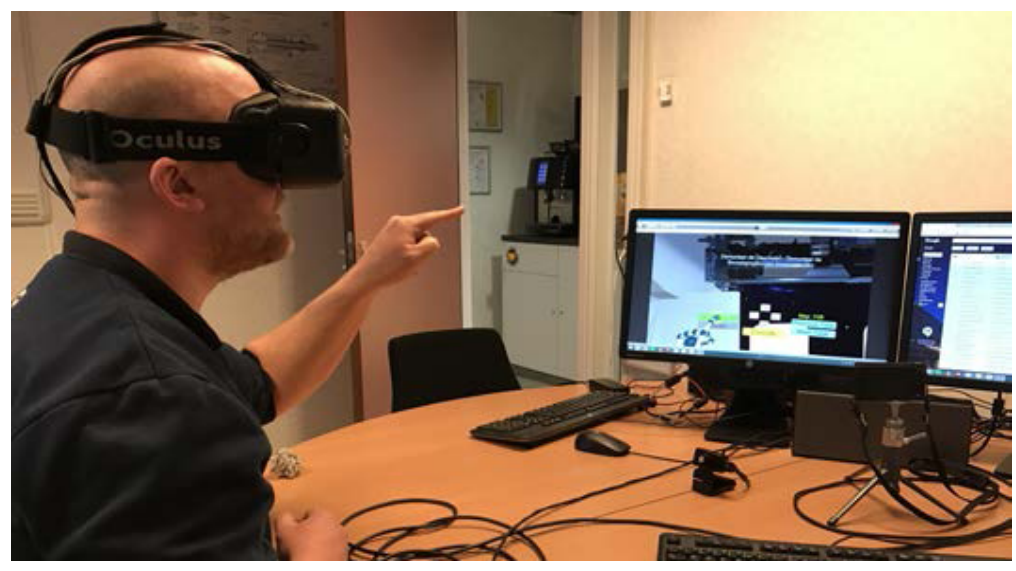

\subsubsection{Limitations of the selected technology}

However, the selected technology and devices present some issues that have to be underlined.

Firstly, due to technological limitations, no tool (wrenches and screwdrivers) are included in the training: nuts and bolts do not need to be loosened or tightened. Even if specifications (i.e., torque and tension) are not needed to be simulated within the VR, the information needs to be transferred to the technicians in order to still have a valuable VMTT. Consequently, these specifications appear at the end of the task descriptions.

Secondly, no spare part is present in the training. Literature studies show that it possible adding a virtual warehouse wherein the technician can prepare for the maintenance procedure (Garcia et al., 2016). However, since only two components within the selected maintenance operation have to be replaced, the learning benefit for the technicians does not outweigh the added effort to create such an environment. Such a feature would be valuable to add in a second phase, after a positive feedback on VMTT by the technicians.

Finally, no wire and cable is displayed in the VR. According to NS experts' reflections, the presence of those parts is not fundamental for reaching the learning objectives. Nevertheless, the power cable is mentioned in the preparation of the task description for highlighting the need of switching off the electricity before working on the door motor.

\subsection{Evaluation of the VMTT}

Since the VMTT is an interaction between the users and the VR environment, the evaluation method shall use principles described in the research field of Human-Computer Interaction (HCI), defined as the study of the way in which computer technology influences human work and activities (Blanton et al., 2009). The quality of 
the instructions, the quality of the VR, the value of the VMTT for technicians, the value of VMTT for the NS, the reduction of the maintenance time with VMTT are some of the aspects to investigate in the evaluation phase.

For those reasons, the chosen evaluation method combines a heuristic evaluation approach, characterised by a designer-user direction, with a cognitive walkthrough technique, where the user can autonomously experience the software and its functions. On the one hand, the presence of a heuristic part permits to identify issues with the User Interface (UI) design regarding the usability and to assess the software's compliance (Nielsen and Molich, 1992). On the other hand, the presence of the cognitive walkthrough underlines the problem learnability process (Polson et al., 1992).

The result is a questionnaire separated into three main sections:

- survey before VMTT

- $\quad$ test after VMTT

- survey after VMTT.

The purpose of the survey questions in each section is two folds: focusing on the key performance indicators (KPIs) related to the user, to the VR and to the company and focussing on some overall indicators defined by the International Organisation for Standardisation (ISO) (ISO:25010:2011, 2011).

The surveys and tests are created with the support of Google Forms. This allows a multitude of different question types and with a pleasing graphical presentation of answers. The survey makes use of short textual answers and ratings (i.e., from 1 to 5 or 1 to 10), based on the Likert scale (Likert, 1932).

\subsubsection{Survey before VMTT}

This questionnaire is proposed before the technician experiences the VMTT. It mainly aims to assess the technician's proficiency and personal attitude with HCI, their motivation in doing the training and expectations accompanied with the VR technology. During the survey personalia are collected as, age, working experience, qualifications as technician and familiarity with FLIRT and Sprinter Light Train (SLT). Their experience with the SLT is also investigated since components and assemblies are similar between the FLIRT and SLT.

\subsubsection{Test after VMTT}

After the experience with the VMTT, a test to quantitatively measure the learning retention is proposed to the technicians. The test has 20 questions:

- 15 questions concern the task instruction (method of certain tasks or the order at which they are to be carried out)

- five questions focus on technical specifications (embedded as information in the VR) for specifically checking the retention knowledge on torque information, component size, or amount of parts. 


\subsubsection{Survey after VMTT}

The last part of the questionnaire is provided to receive a structured feedback from the technicians on the educational capabilities of VMTT, on the quality of the VR, on the quality of the instructions, on the efficiency, utility, practicality, and applicability of the VMTT and on personal future expectations for the extensive use of the VMTT.

Having a bottom-up approval for these innovative training technologies is vital for the NS in order to achieve a shared acceptation amongst the technicians of the new method for ensuring valuable results for the quality of the maintenance operations.

\section{Part 3 - virtual maintenance task training}

To achieve good results, the VMTT has to be technically accurate, intuitive to use for quick deployment and educationally relevant. Consequently, the design of the VR requires particular attention in every step, from the development of the VR to the identification of the task instructions.

Figure 6 View of the training in the VMTT (see online version for colours)

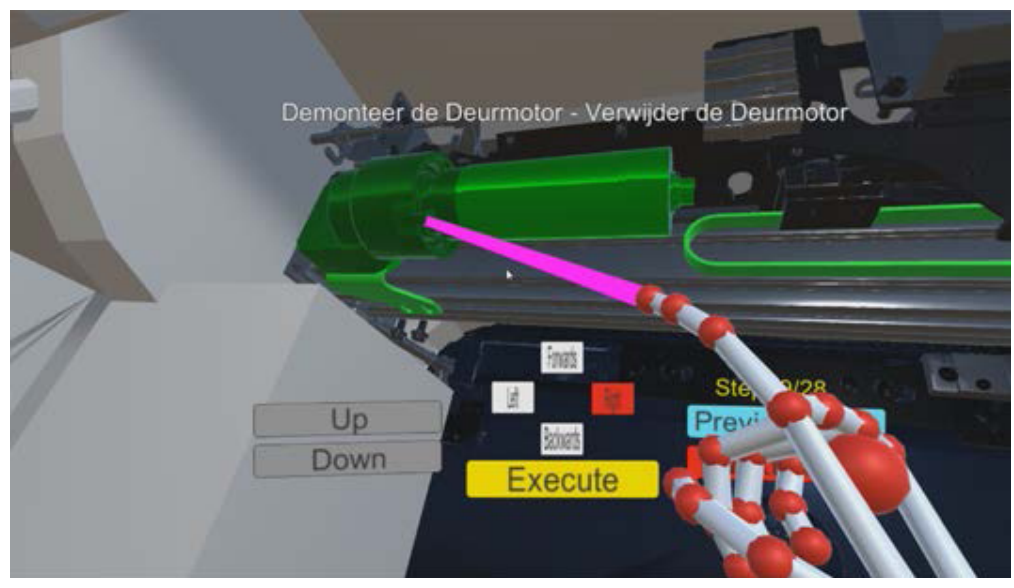

Note: The green part is the component selected by the technician to be removed.

The VMTT starts after the technician wears the HMD. The VR environment is loaded, and the technician is transported into the virtual FLIRT besides the door. Raising his/her hands starts the VMTT. The maintenance task is described at the top of his/her field of view (Figure 6). By using his/her hands, he/she can translate the character through the scene. With the head movements, the technician can look inside the mechanisms of the door.

The displayed buttons in the VR environment are operated by the technician using hands and fingers. The Leap Motion detects them and within the VMTT the hand fulfils the control functions.

Furthermore, the right hand has an extra property. From its index finger a ray is cast which can correctly highlight the selected parts. By pointing, in real life, the virtual character follows and aims at the digital parts. 
The task descriptions guide the technician towards the relevant components. When the component is selected at the correct step, it becomes highlighted with the green colour. Then, it is possible to (dis)assemble the component. If the technician continues to follow the described tasks, he/she can complete the training and replace the door motor of the FLIRT door.

\subsection{Developing VR}

The VR environment is realised from CAD files. They provide the technical components for the environment, within a parametric modelling program as Autodesk or Solidworks. Through a conversion from .3DXML (3D file format), all the design information is transferred in .OBJ files (containing the $3 \mathrm{D}$ geometry) for further digital optimisation processes.

The resulting optimised .OBJ files for the door system and wagon are combined and imported into Unity3D (3D modelling software) where the environment is created with controllable characters operating through C\# scripts. Figure 7 shows some steps of the creation process for the door motor and for the train couch.

Figure 7 Some steps of the creation of the VR (see online version for colours)

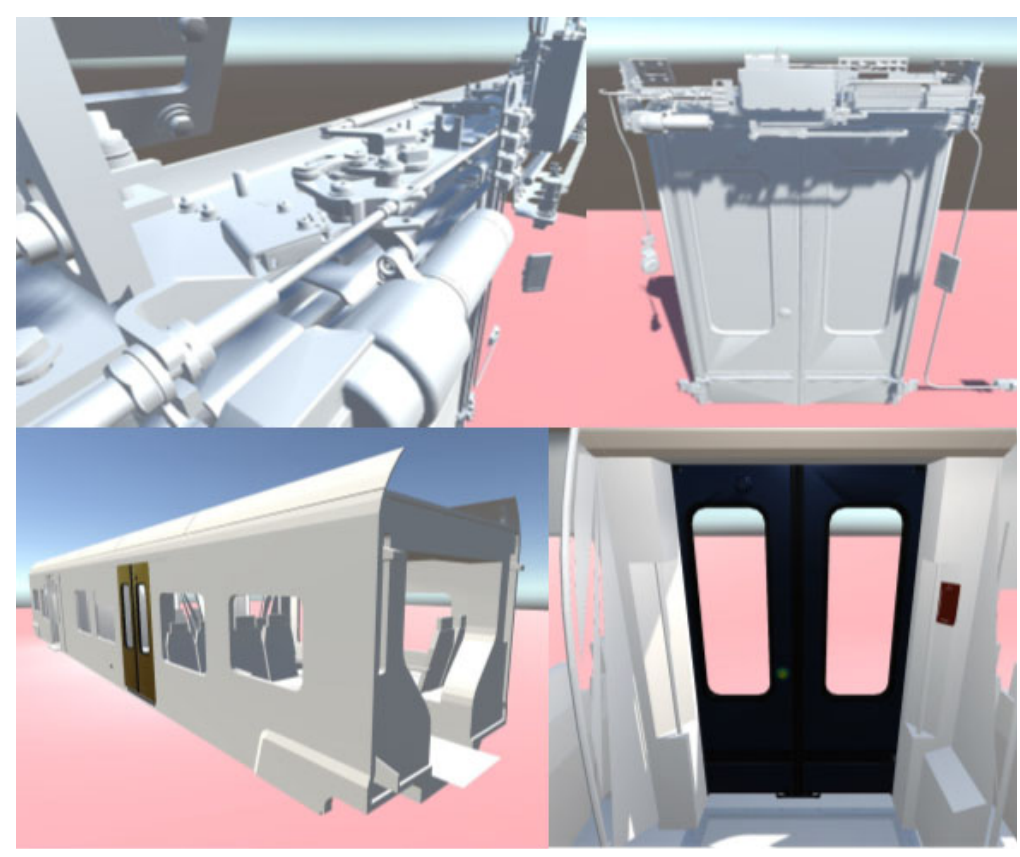

\subsection{Task instruction}

Since the working instructions for assembling/disassembling the door motor are not entirely translatable within the VR environment, some adjustments and simplifications (tasks' combination) are needed: the door is already removed from power, the hatch is opened, and the power plug is removed from the door motor. The only required action is 
opening the hatch to get access to the door mechanism. The same simplification has been applied for closing the hatch and connecting the door motor to power.

The tasks and its instructions are shown in Figure 8. The procedure is formed by 29 tasks in total.

Figure 8 An overview of the maintenance tasks to replace the door motor (see online version for colours)

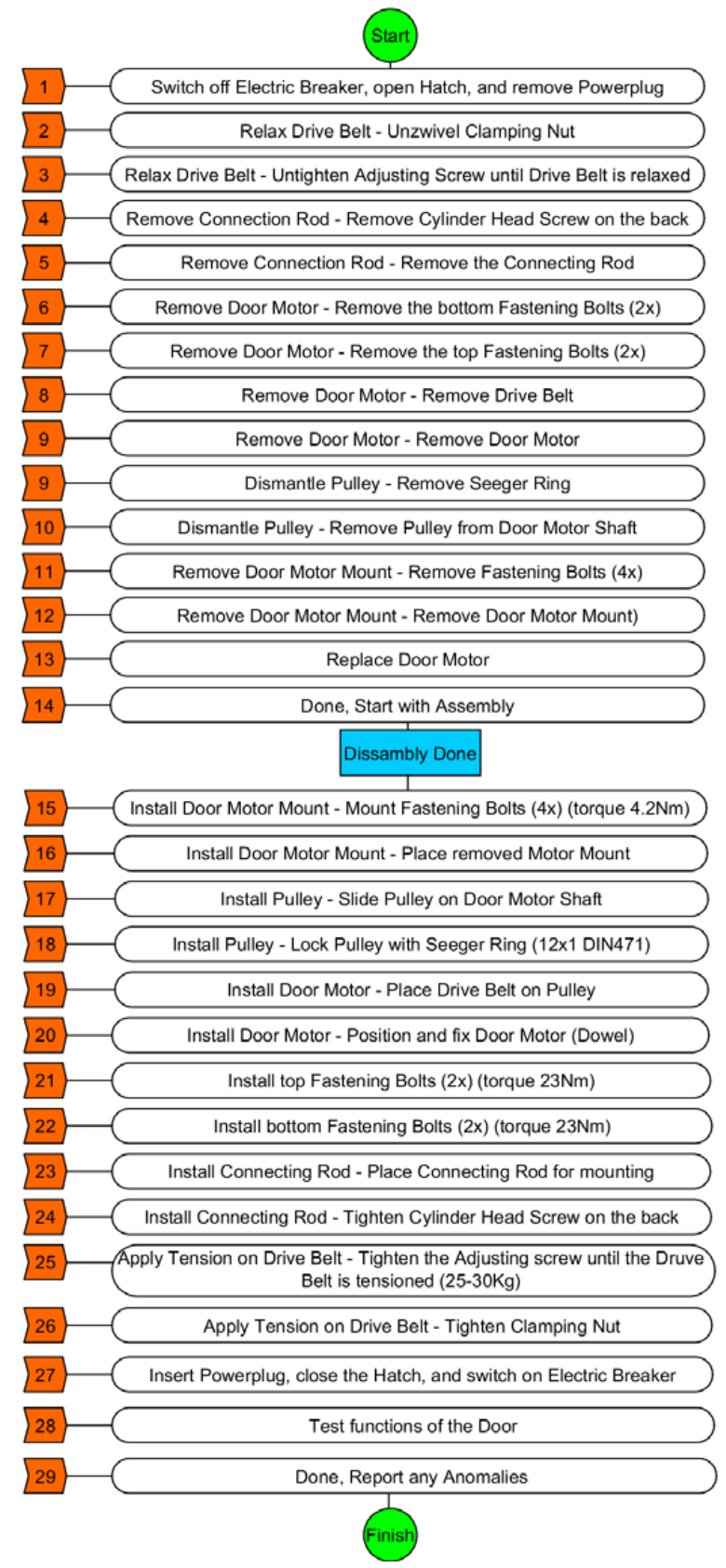




\section{Part 4 - testing phase}

The performed tests are the first chance for the technicians to experience VMTT and in most cases to experience VR as well. For them, it is an opportunity to try this new technology, to learn and memorise the maintenance procedure and to familiarise with the locations of each component in a play like manner. For the NS, it is an opportunity to explore the technician's perspective towards the application of new technologies for maintenance training.

\subsection{Target group and test set-up}

The testing phase took place at the NS service location in Maastricht, where the FLIRTs are initially maintained. In total 23 technicians and practice instructors experienced the VMTT. On average, they have worked for the NS for 14 years; the most experienced technician has been there for 38 years, the less experienced for one year. Figure 9 shows the age repartition and their pre-existing knowledge on FLIRT and SLT trains. The test required an hour for each technician.

Figure 9 (a) The ages of the technicians expressed in years (b) The technician's familiarity with SLT and FLIRT trains (see online version for colours)

Age

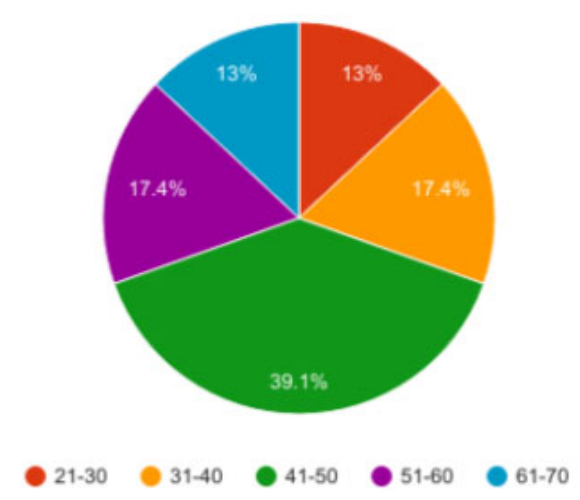

(a)

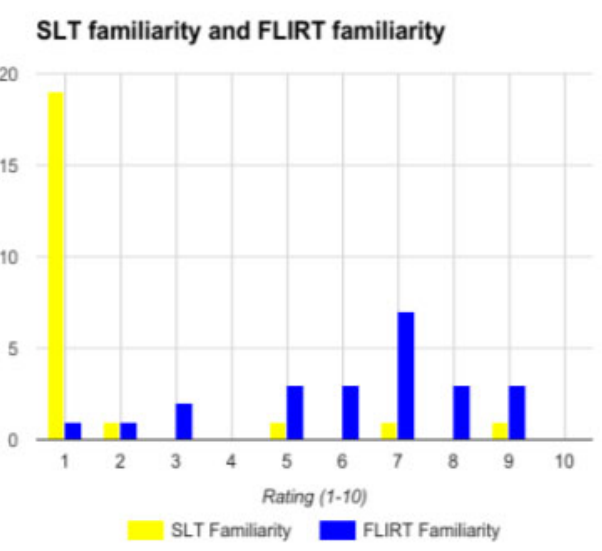

(b)

Since, as mentioned before, for most of the technicians (70\%) the test was the first time they experienced the basics of VMTT or VR, a first and simple VR environment was setup to let them become familiar with the device and with the technology. In this environment, the technician had to move a cube and look around to understand how the movements were represented. This experience took about five minutes to perform the necessary actions.

\subsection{Results of the testing phase}

As already explained, the aim of the testing phase was to assess not only the quality of the technology adopted (Figure 10) but also the effectiveness of the new training method. 
The technicians had to evaluate the KPIs such as usefulness of the VMTT, effectivity of the VMTT compared to traditional work instructions, benefit of the VMTT, value of VMTT for technicians and value of VMTT for the NS (Figure 11).

Figure 10 Technician's rating of quality related to the instructions, control, HMD, VR and immersion after VMTT (see online version for colours)

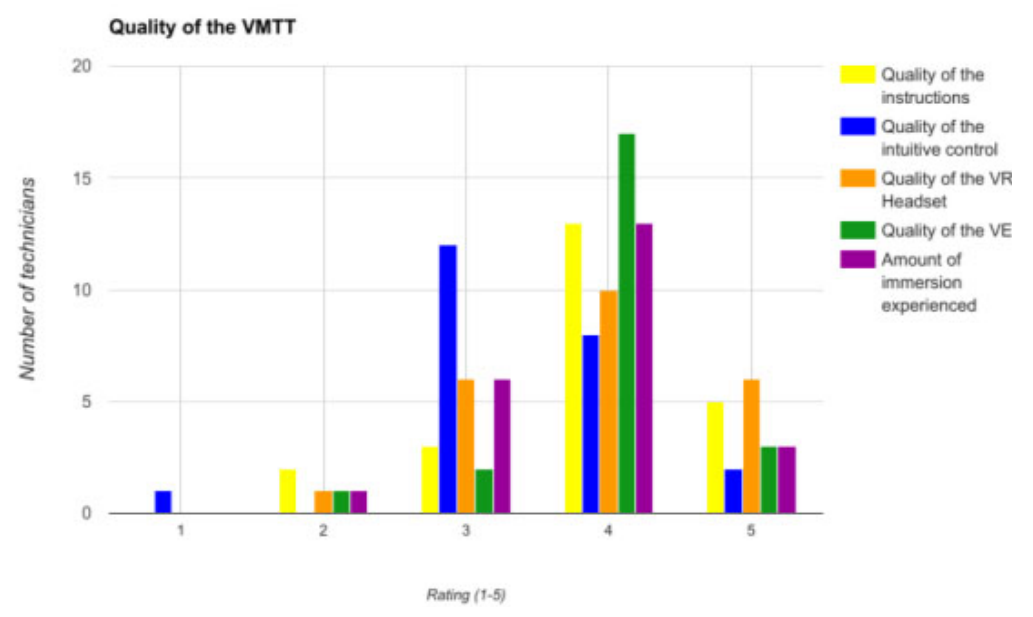

From the feedback collected through the surveys, it appears that the FLIRT and the components in the VMTT have an adequate detail level for training. The technicians generally appreciated the VR environment even though some maintenance procedures contained small inaccuracies and few tasks would have been ordered differently in real life.

\section{Discussion and conclusions}

In general, virtual learning introduces interesting possibilities for the improvement of the maintenance training. When correctly designed, the technician can train and study components of trains without the need for the asset, classrooms, or practice instructors. A complete virtual learning system can instruct and evaluate the technicians' knowledge. This definitely increases the effectiveness of a traditional training.

Moreover, as proved by the VMTT (Figure 11), the distance between education and operation is decreased.

Virtual learning transfers knowledge more effectively and has proven to directly apply it to real life skills. The technicians are open and ready to a virtual learning method to support them during training. Moreover, they appreciated the opportunity offered by the VR environment of visualising components of the motor impossible to see in the normal training.

Even though the use of VR was beneficial to the immersion of the technician still some improvements are needed to properly educate them and in the current stage the VMTT can not replace traditional training but can only support it. 
Moreover, to build a complete Virtual Educational System applicable and flexible to all train series, some changes in the company structure are needed. A possible solution is proposed in Figure 12.

Figure 11 The technician's rating on the overall quality of VMTT and the potential for VMTT (see online version for colours)

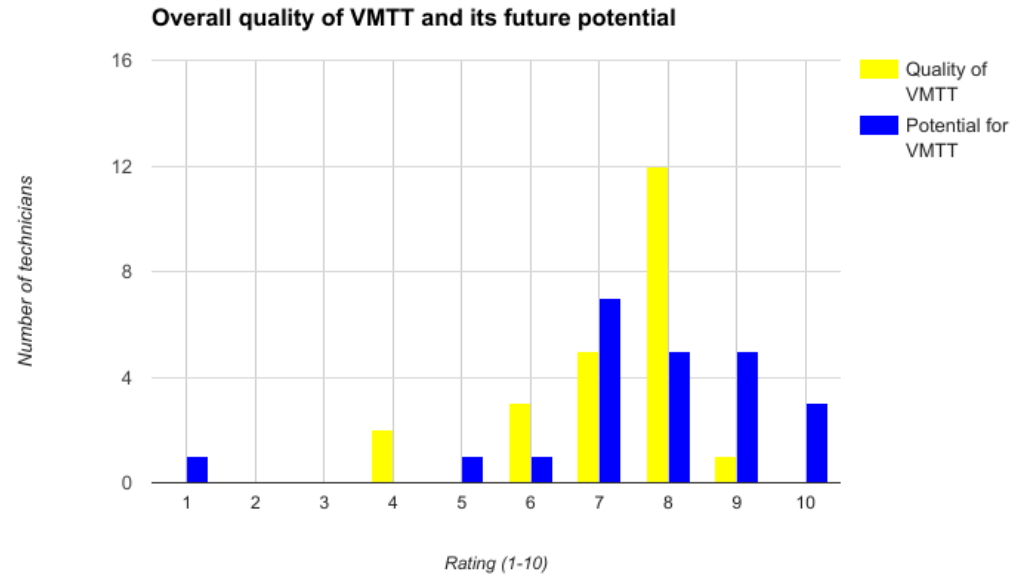

Figure 12 An overview of a possible company structure for fully embracing virtual training on a large scale (see online version for colours)

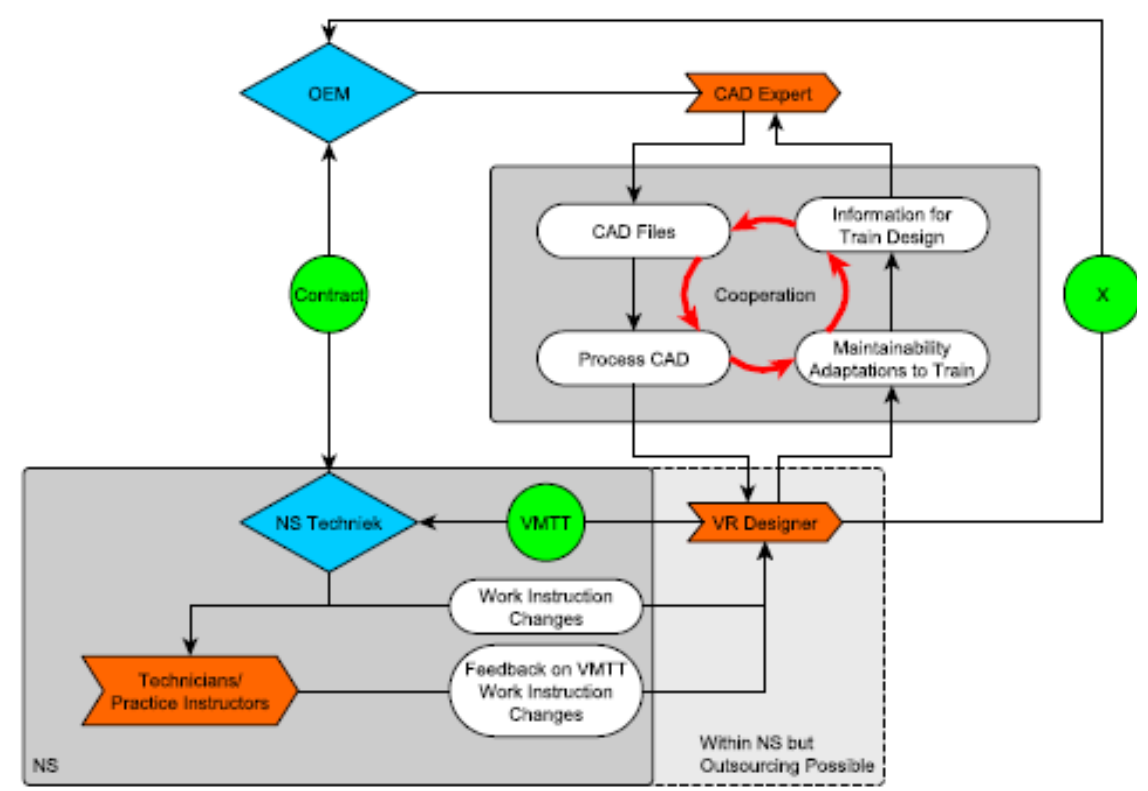

A job function, as VR designer, should be created focused on the VMTT. He/she should work together in close cooperation with the CAD expert of the OEM. They create, maintain, and adapt the VMTT based on changing work instructions, maintenance procedures, and feedback from technicians, reliability engineers, and maintenance 
engineers. Their connection is described as a circular process where the CAD expert delivers files and receives information relevant for train design adaptations. The VR designer receives compatibles CAD files ready for processing. This close cooperation permits to save resources and working hours in the process. He/she provides maintainability adaptations for the train.

Those adaptations come from technicians' feedback and practice instructions but also from the changing work instructions made by maintenance and reliability engineers of the NS. The VR designer delivers it to the NS, which applies it within their education system.

Concluding, the research should be continued in the direction of virtual maintenance training. Technicians and practice instructors of the NS were supportive and willing to allow a solution in their day-to-day operations.

Next steps of research should consider investigations in visualisation (exploring the possibilities of different HMDs, different sensors, and improved VR quality), interaction (new VR systems for implementing player movement and allowing the technicians to explore the virtual train by mimicking the movements in real life as jumping and ducking), and evaluation (creating a standardised framework to evaluate traditional learning versus virtual learning methods).

\section{Acknowledgements}

The presented paper was made achievable with the precious contribution in terms of data, technicians, experts and resources provided by Netherlands Railways (NS).

\section{References}

Bamodu, O. and Ye, X. (2013) 'Virtual reality and virtual reality system components', Proceedings of the 2nd International Conference on Systems Engineering and Modeling, pp.921-924.

Blanton, M., Zhou, J., Moro, M.M., Zhang, D. Tsotras, V.J., Halkidi, M., Hainaut, J.L., Tanin, E., Korn, F.R., Pedersen, T.B., Bohm, C., Plant, C., Zhang, Q., Strauss, M.J., Jensen, C.S., Snodgrass, R., Li, N., Kantarcioglu, M., Sebe, N., Jaimes, A., Dix, A. and Tompa, F.W. (2009) 'Human-computer interaction', in: Encyclopedia of Database Systems, pp.1327-1331, Springer US, Boston, MA.

Bonk, C.J., Graham, C.R., Cross, J. and Moore, M.G. (2012) The Handbook of Blended Learning: Global Perspectives, Local Designs, Pfeiffer Essential Resources For Training and HR Professionals, Wiley, San Francisco, CA.

Chomko, R. (2012) Maintaining an App is Critical to its Overall Success [online] https://www.fiercewireless.com/developer/maintaining-app-critical-to-its-overall-success.

Ebbinghaus, H. (2013) 'Memory: a contribution to experimental psychology', Annals of Neurosciences, Vol. 20, No. 4, pp.55-156.

European Federation of National Maintenance Societies (EFNMS) (1998) The Requirements and Rules to Achieve an EFNMS Certificate as a European Expert in Maintenance Management, Technical Report, May.

Garcia, A.A., Bobadilla, I.G., Figueroa, G.A., Ramirez, M.P. and Roman, J.M. (2016) 'Virtual reality training system for maintenance and operation of high-voltage overhead power lines', Virtual Reality, Vol. 20, No. 1, pp.27-40.

International Standards Organisation - ISO:25010:2011 (2011) Systems and Software Engineering - Systems and Software Quality Requirements and Evaluation (SQuaRE) - System and software quality models, Technical Report ISO 25010:2011, ISO/IEC. 
Likert, R. (1932) 'A technique for the measurement of attitudes', Archives of Psychology, Vol. 22, pp.140-155.

Martinetti, A., Rajabalinejad, M. and van Dongen, L.A.M. (2017) 'Shaping the future maintenance operations: reflections on the adoptions of augmented reality through problems and opportunities', Procedia CIRP 59, 14-17, Springer International Publishing, pp.29-55. ISBN:978-3319-12110-9.

Nielsen, J. and Molich, R. (1992) 'Heuristic evaluation of user interfaces', CHI '90 Proceedings of the SIGCHI Conference on Human Factors in Computing Systems, pp.249-256.

Pintelon, L. and Parodi-Herz, A. (2008) 'Maintenance: an evolutionary perspective', in: Springer Series in Reliability Engineering, Vol. 8, pp.21-48, Springer London, London.

Polson, P.G., Lewis, C., Rieman, J. and Wharton, C. (1992) 'Cognitive walkthroughs: a method for theory-based evaluation of user interfaces', International, Journal of Man-Machine Studies, Vol. 36, No. 5, pp.41-773.

Rajabalinejad, M., Martinetti, A. and van Dongen, L.A.M. (2016) 'Operation, safety and human: critical factors for the success of railway transportation', IEEE 11th International Conference on System of Systems Engineering (SoSE 2016), 12-16 June, Kongsberg, Norway.

Salas, E., Tannenbaum, S.I., Kraiger, K. and Smith-Jentsch, K.A. (2012) 'Commentary on the science of training and development in organizations: what matters in practice', Psychological Science in the Public Interest, Vol. 13, No. 2, pp.73-101.

Stadler Bussnang AG (2016) Stadler FLIRT 3 EMU - Maintenance Manual, Technical Report, Bussnang. 